\title{
Subcycle control of the photoelectron angular distribution using two-color laser fields having different kinds of polarization
}

\author{
Xufei Sun, ${ }^{1,2, *}$ Denhi Martinez $\odot,{ }^{1}$ Patrick Froß, ${ }^{1}$ Nicolas Camus, ${ }^{1}$ Yonghao Mi $\odot,{ }^{1}$ Weiyu Zhang $\odot,{ }^{1}$ \\ Zhanghai Chen, ${ }^{2}$ Thomas Pfeifer, ${ }^{1}$ and Robert Moshammer ${ }^{1, \dagger}$ \\ ${ }^{1}$ Max-Planck-Institut für Kernphysik, Saupfercheckweg 1, 69117 Heidelberg, Germany \\ ${ }^{2}$ Department of Physics, Xiamen University, Xiamen 361005, China
}

(Received 7 December 2020; accepted 23 February 2021; published 8 March 2021)

\begin{abstract}
We investigate the subcycle control of electron trajectories in single ionization of Ar atoms with two-color laser pulses consisting of a linearly polarized $800-\mathrm{nm}$ field and a circularly polarized $400-\mathrm{nm}$ field. By varying the relative phase between the two fields, the photoelectron angular distribution rotates in the polarization plane and the rotation velocity can be controlled. From the comparison with results obtained with a semiclassical model, we find that the Coulomb field has a greater impact on direct trajectories than on those that undergo a recollision which is opposite to the electron behavior in a monochromatic field. This effect can be directly visualized in the experiment and finely controlled on a subcycle timescale by means of the two-color field scheme. It is shown that the influence of the Coulomb force on the photoelectron momentum distribution is different along the longitudinal and transverse direction.
\end{abstract}

DOI: 10.1103/PhysRevA.103.033106

\section{INTRODUCTION}

In laser-driven light-atom interactions a valence electron is photoionized on an attosecond timescale. This ultrafast electron dynamics has attracted an abundance of attention because it is one of the most fundamental processes in light-matter interactions, and it gives rise to many new exciting phenomena [1]. Owing to the rapid advancements in laser technology, the control of electron motion in atoms and molecules has become possible and has thus been intensively investigated in the past two decades. Carrier envelope phase stabilized few-cycle pulses [2-8], single subfemtosecond pulses [9-11], and near-infrared femtosecond pulses combined with synchronized extreme ultraviolet attosecond pulses [12-15] are efficient tools to successfully control the ultrafast electron dynamics in strong-field ionization.

In recent years, two-color laser fields have been widely used to control the photoelectron motion on a subcycle timescale. In many cases, these fields consist of the fundamental wave $(\mathrm{FW})$ and its second harmonic $(\mathrm{SH})$ component. With the well-defined relative phase between them, the photoelectron ionization dynamics can be finely controlled. Using parallel two-color (PTC) laser pulses, one can control the photoelectron interference pattern $[16,17]$, the photoelectron

\footnotetext{
*xfsun@xmu.edu.cn

${ }^{\dagger}$ robert.moshammer@mpi-hd.mpg.de
}

Published by the American Physical Society under the terms of the Creative Commons Attribution 4.0 International license. Further distribution of this work must maintain attribution to the author(s) and the published article's title, journal citation, and DOI. Open access publication funded by the Max Planck Society. angular asymmetry $[18,19]$, and the asymmetric dissociation of molecules [20-23]. With orthogonally polarized two-color (OTC) laser fields the photoelectron momentum distributions can be controlled even in two dimensions [24]. Moreover, subcycle control of the inter- and intracycle interferences in photoelectron emission, the control of electron-electron correlation in double ionization [25,26], and the directional emission of nuclear fragments $[27,28]$ in ionization of molecules have been achieved. Utilizing two-color circularly polarized fields with different helicity trefoil and semilunar patterns of proton emission have been observed [29], and by means of two-color corotating circular fields a double-pointer attoclock photoelectron interferometer was produced [30]. Recently, a $45^{\circ}$ cross linearly polarized twocolor (CTC) laser field was employed to study the effect of Coulomb focusing and defocusing on the photoelectron motion by tuning the relative phase between the constituent laser fields [31]. In all previous works, mainly two-color fields with the same type of polarization (only bilinear or bicircular) were combined. Here, we employ two laser fields with different colors and different polarization (linear and circular) to study and control the electron dynamics in photoionization.

The parent ion's Coulomb potential has manifested its importance in modifying the trajectory of the tunneled electron in many experiments and numerical simulations. The trajectories of the recolliding electrons are always most affected because of their return to the parent ion. Recently, a surprisingly strong distortion of direct, nonrecollision trajectories has been verified in an OTC field [24]. However, since the contributions from recollision and nonrecollision trajectories are still coupled in the momentum spectrum, this effect cannot be observed directly in the experiment. Here, we show that this peculiar effect can be clearly visualized and precisely 

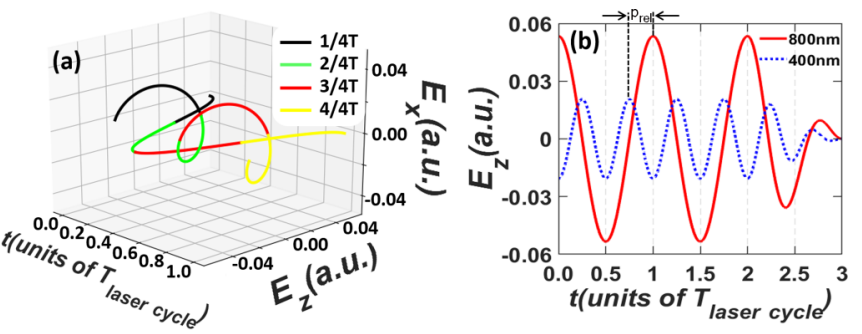

FIG. 1. (a) Illustration of the two-color electric field generated with linearly polarized $800-\mathrm{nm}$ and circularly polarized 400-nm laser pulses in one cycle for $p_{\text {rel }}=\pi$. Four colors denote four quarter cycles of the 800-nm laser field. (b) 800-nm (solid red line) and 400-nm (dotted blue line) electric fields along the $z$ direction for $p_{\text {rel }}=\pi$. The intensities of the 800 - and $400-\mathrm{nm}$ fields are $1.0 \times 10^{14} \mathrm{~W} / \mathrm{cm}^{2}$ and $0.3 \times 10^{14} \mathrm{~W} / \mathrm{cm}^{2}$, respectively.

controlled by means of a two-color laser field having two different types of polarization.

In this work, we measured the photoelectron angular distribution (PAD) of argon atoms in a two-color laser field which combines a linearly polarized $800-\mathrm{nm}$ field with a circularly polarized field with doubled frequency. By varying the relative phase between them, we can control the PAD in the polarization plane. The PAD rotates in the plane of polarization with increasing relative phase, but not at a constant speed. By comparing with the results of classical trajectory Monte Carlo (CTMC) calculation, we study the influence of the parent ion's Coulomb force on the PAD. After separating the phase-dependent PAD into contributions emerging from different electron birth times we find that direct trajectories are suppressed and distorted more than the recollision trajectories. This effect can be directly visualized and controlled by tuning the relative phase in the experiment. The phase-dependent momentum spectra show further that the Coulomb interaction has an opposite effect on the photoelectron longitudinal and transverse momentum distributions.

\section{CLASSICAL PREDICTION}

The laser field used in the calculations consists of a linearly polarized $800-\mathrm{nm}$ field and a circularly polarized $400-\mathrm{nm}$ field. The polarization plane is the $z-x$ plane and the $800-\mathrm{nm}$ field is polarized along the $z$ axis. Figure 1(a) shows the individual components of the electric field over one laser cycle. Here, the ratio of the intensity of the $400-\mathrm{nm}$ field to the $800-\mathrm{nm}$ field is 0.3 . The electric field of this two-color pulse can be expressed as

$$
\begin{aligned}
\vec{E}(t)= & f_{z}(t)\left[E_{0, R} \cos \left(\omega_{R} t\right)+\frac{E_{0, B}}{\sqrt{2}} \cos \left(\omega_{B} t+p_{r e l}\right)\right] \vec{e}_{z} \\
& -f_{x}(t) \frac{E_{0, B}}{\sqrt{2}} \sin \left(\omega_{B} t+p_{r e l}\right) \vec{e}_{x}
\end{aligned}
$$

where $E_{0, R}\left(\omega_{R}\right)$ and $E_{0, B}\left(\omega_{B}\right)$ are the electric field amplitude (frequency) of the 800- and 400-nm fields, respectively; $f_{z, x}$ denote the pulse envelope along the $z$ and $x$ directions, respectively; designates the relative phase between the two colors as shown in Fig. 1(b). Atomic units (a.u.) are used throughout unless specified.

With this two-color laser field we first use a classical trajectory model to predict the electron momentum distribution at different relative phases, as shown in Fig. 2(a). In this simplest strong-field ionization model the electron momentum distribution is obtained by a classical time to momentum mapping relation $\vec{p}=-\vec{A}\left(t_{0}\right)$ with a probability determined by the ionization rate at the spatial origin [32] and a narrow Gaussian-like momentum distribution around the final electron momentum $\vec{p}$. Here, $\vec{A}(t)=-\int_{-\infty}^{t} \vec{E}\left(t^{\prime}\right) d t^{\prime}$ is the vector potential and $t_{0}$ is the electron birth time. Since we ignore the Coulomb force the electrons are driven only by the two-color laser field and sorted into different momentum regions in the $z-x$ plane according to their birth time. One can find that the PAD is always mainly distributed in adjacent quarter cycles rather than two quarter cycles apart and it rotates anticlockwise with the increasing two-color $p_{\text {rel }}$. This characteristic behavior and the rotation of the PAD are
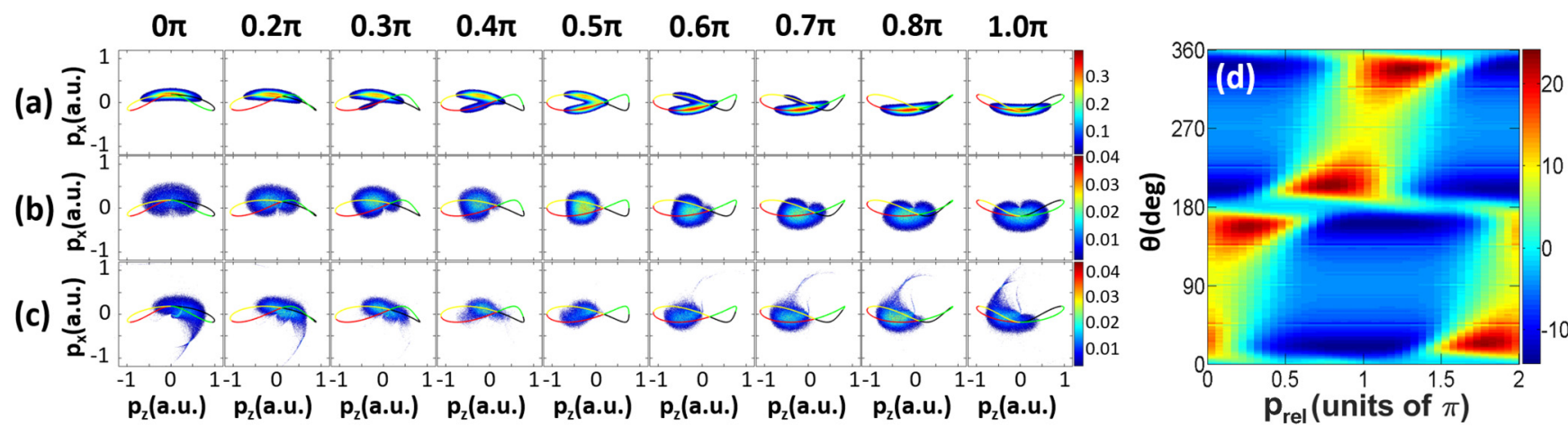

FIG. 2. (a)-(c) Photoelectron momentum distribution in the $z-x$ polarization plane for various $p_{\text {rel }}$ simulated by the classical trajectory model (a), and CTMC model without (b) and with (c) inclusion of the Coulomb potential. The distribution from $\pi$ to $2 \pi(0)$ is central symmetrical of the distribution from 0 to $\pi$. The field-driven electron momentum curve $\vec{p}=-\vec{A}\left(t_{0}\right)$ for the specific two-color field is drawn on top of the momentum distributions. The colors encode quarter cycles of the 800-nm field. (d) The PAD with respect to the $p_{\text {rel }}$ calculated by the classical trajectory model. 


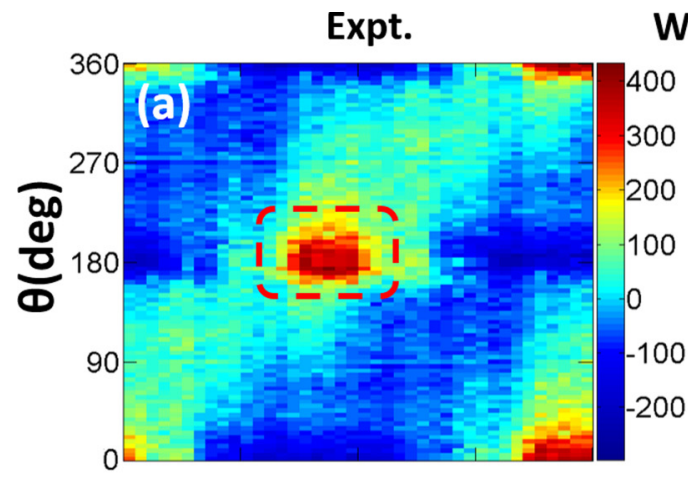

Without Coulomb potential included
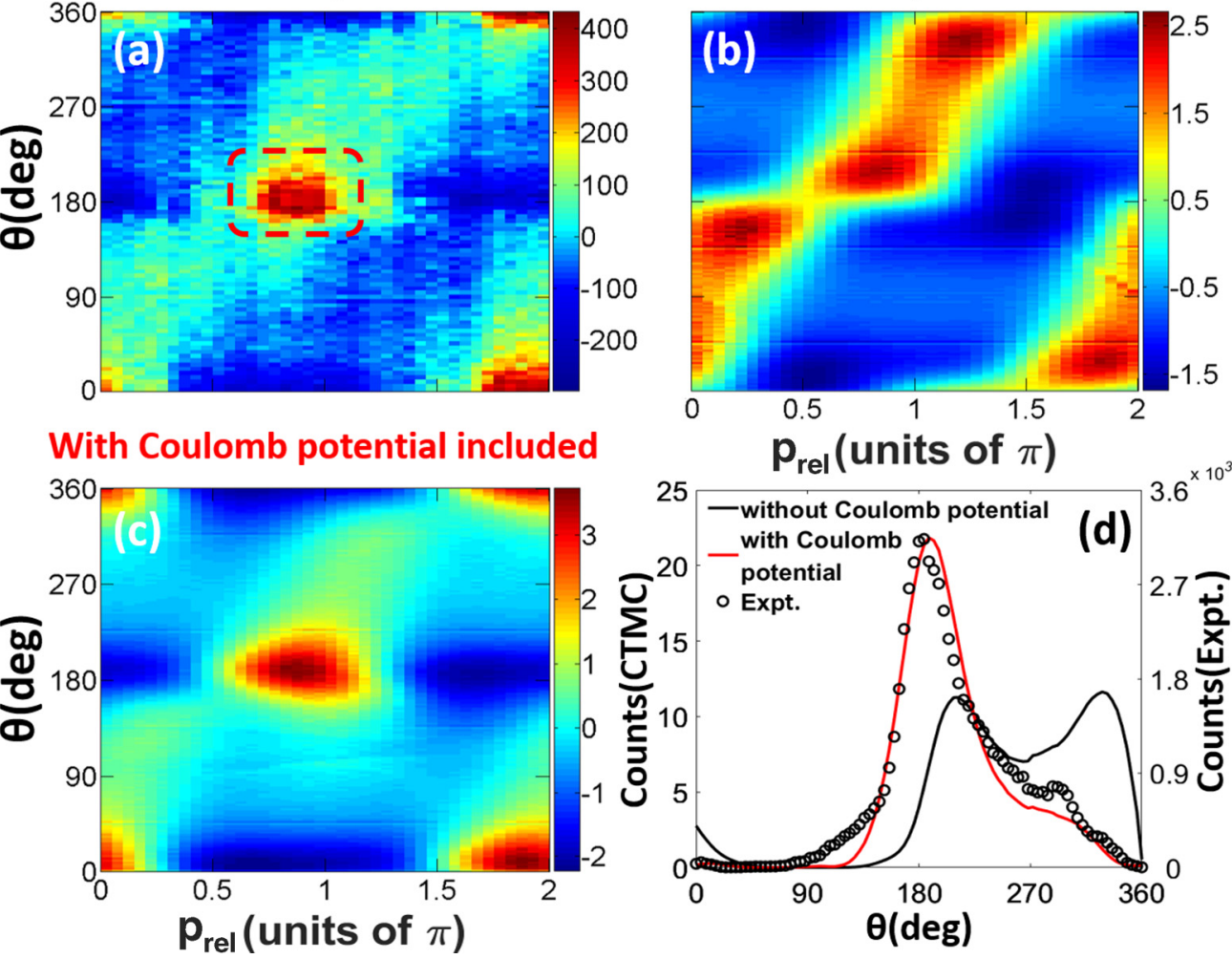

FIG. 3. The measured (a) and simulated (b), (c) PADs with respect to the $p_{r e l}$. (b), (c) CTMC simulations without (b) and with (c) the parent ion's Coulomb potential included. (d) The measured (open circles) and simulated (solid line) photoelectron emission angle distribution after integrating over the two-color relative phase $p_{\text {rel }}$ in the range from $0.5 \pi$ to $1.5 \pi$. The black and red lines represent the results without and with inclusion of the Coulomb potential, respectively.

determined by the composite field. To achieve a more straightforward observation of this $p_{\text {rel }}$-dependent rotation effect, and to increase the overall visibility, we plot the electron yield as a function of the electron emission angle and the phase [Fig. 2(d)]. We deal with the photoelectron momentum distribution in the $z-x$ plane as $Y N_{p_{r e l, i}}\left(p_{z}, p_{x}\right)=Y_{p_{\text {rel }, i}}\left(p_{z}, p_{x}\right)-$ $(1 / N)\left[\sum_{p_{r e l, i}=0}^{p_{r e l}=2 \pi} Y_{p_{r e l, i}}\left(p_{z}, p_{x}\right)\right]$, where $Y_{p_{r e l, i}}\left(p_{z}, p_{x}\right)$ is the $p_{\text {rel }, i}$-dependent electron yield in the $p_{z}-p_{x}$ momentum plane, and $N$ is the total number of scanning steps over the $p_{\text {rel }}$ phase. Then, after momentum integration along the electron emission angle $\theta$, where $\theta=\cos ^{-1}\left(p_{z} / \sqrt{p_{z}^{2}+p_{x}^{2}}\right)$, we get two separated, tilted sandglasslike structures in the $p_{\text {rel }}$-dependent electron angular distribution, as shown in Fig. 2(d).

\section{EXPERIMENTAL SETUP AND EXPERIMENTAL RESULTS}

In our experiment the two-color laser pulses with different polarizations were produced using a Mach-Zehnder interferometer. The linearly polarized FW was generated with a Ti:sapphire multipass amplifier (25 fs, $800 \mathrm{~nm}, 3 \mathrm{kHz}$ ). After creating a copy with a beam splitter, one of the beams was frequency doubled by a $200 \mu \mathrm{m}$ thick $\beta$-barium borate crystal $(\beta$-BBO $)$ to obtain a $\mathrm{SH}$ pulse. A quarter-wave plate was put right after the $\mathrm{BBO}$ crystal to produce the circularly polarized $\mathrm{SH}$ field. The $p_{r e l}$ delay between the two colors was controlled by a motorized translation delay stage in the $\mathrm{SH}$ arm of the interferometer. The two-color laser pulses were focused onto a supersonic gas jet of Ar atoms by a concave mirror inside a reaction microscope. Then, correlated ion and electron fragments were measured by cold-target recoil-ion reaction momentum spectroscopy (COLTRIMS) [33]. The peak intensity of FW and $\mathrm{SH}$ was estimated to be $(1.0 \pm 0.1) \times$ $10^{14} \mathrm{~W} / \mathrm{cm}^{2}$ and $(0.3 \pm 0.1) \times 10^{14} \mathrm{~W} / \mathrm{cm}^{2}$, respectively. The absolute relative phase between the two laser fields was calibrated by comparison with the numerical results.

Figure 3(a) shows the measured PAD versus the two-color $p_{\text {rel }}$. The shape of the spectrum still exhibits an antidiagonal distribution in the $2 \pi p_{\text {rel }}$ range which means the PAD rotates anticlockwise with increasing $p_{\text {rel }}$. However, the experimental and classical results [see Figs. 3(a) and 2(d)] do not correspond to each other in many details. Firstly, the tilted sandglasslike structure is only barely visible in the experiment which exhibits pronounced yield maxima around $p_{\text {rel }} \approx 0.87 \pi$ and $1.87 \pi$. Secondly, in comparison with the experimental result, the classical spectrum shows clear yield minima or valleys in the distribution around $\left(0.5 \pi, 180^{\circ}\right)$ and $\left(1.5 \pi, 360^{\circ}\right)$. But the rotation velocity jump in these two regions is predicted by the classical model. This means the nonuniform rotation of the PAD is entirely caused by the twocolor laser field. From the electron momentum distribution and field-driven momentum curve shown in Fig. 2(a), we can find that at most of the relative phases, the field-driven momentum curves of the first (third) and second (fourth) quarter 
cycles are always close to each other, but their ionization rates are totally different except around $p_{\text {rel }}=0.5 \pi$ and $1.5 \pi$. This results in a dominant angular distribution only pointing to a certain angle with highest ionization probability at most of the relative phases. However, the contributions from both the third (first) and fourth (second) quarter cycles are dominant when $p_{\text {rel }}$ is near $0.5 \pi(1.5 \pi)$, and they are even exactly the same when $p_{\text {rel }}=0.5 \pi(1.5 \pi)$. Therefore, the rotation velocity will decrease around these two relative phases because of the two dominant distributions which are close to each other.

\section{THEORETICAL SIMULATIONS AND DISCUSSIONS}

In order to explore the reasons for the noncorrespondence between the experimental and classical results, we performed a three-dimensional CTMC simulation. In the CTMC model, the electron tunneling exit is derived from Landau's effective potential theory [34]. At the tunneling exit, the electron has a Gaussian-like initial transverse momentum distribution perpendicular to the instantaneous two-color field and a zero longitudinal momentum distribution along it. The electric field dependent ionization rate is given by the ADK theory [35,36]. After tunneling, the evolution of the electron trajectory is obtained by numerically solving the classical equation of motion $d^{2} \vec{r}(t) / d t^{2}=-\vec{E}(t)-\vec{\nabla} V(r)$, where $V(r)=-1 / r$, is the atomic potential ( $r$ is the distance between electron and nucleus). The two-color electric field is given by Eq. (1), where $\omega_{R}=0.057$ a.u. and $E_{0, R}=$ 0.0534 a.u. In order to mimic the experimental laser conditions, we use a half-trapezoidal field envelope as shown in Fig. 1(b), where the amplitudes of the first six cycles are constant and the pulse ramps off to zero during the last four cycles. In addition, the calculations are done for 40 different $p_{\text {rel }}$ values covering the range from 0 to $2 \pi$ in order to achieve a sufficient resolution with affordable computing time.

We first show the CTMC result without inclusion of the Coulomb field, as illustrated in Fig. 3(b). The spectrum essentially resembles our classical result [Fig. 2(d)], as expected, and some features of the experiment [Fig. 3(a)]. The velocity jump is also very clear around $\left(0.5 \pi, 180^{\circ}\right)$ and $\left(1.5 \pi, 360^{\circ}\right)$. Interestingly, the yield minima (or valleys) in these two regions for the classical result are refilled. This is because there is a strong coupling of the electron momenta along the $z$ axis around these two relative phases, and hence the two separate dominant angular distributions [see Fig. 2(a)] converge toward the $z$ axis, as shown in Fig. 2(b). In addition, compared with the classical result, there is more yield around $\left(0,90^{\circ}\right)$ and $\left(\pi, 270^{\circ}\right)$, which agrees with the measurement. This is caused by the initial transverse momentum spread that is included in the CTMC model. With increasing initial transverse momentum spread, the angular distribution will be more prominent in these regions. However, as for the classical spectrum, it does not reproduce the experimental result at all relative phases very well, especially the yield around $\left(0.25 \pi, 170^{\circ}\right)$ and $\left(1.25 \pi, 350^{\circ}\right)$. Therefore, we performed another CTMC simulation which includes the Coulomb potential. The result, as shown in Fig. 3(c), is in a good agreement with the measured spectrum, not only in terms of the nonuniform rotation effect, but also in the electron yield at all relative phases. The electron momentum distribution at various relative phases without and with inclusion of the ionic potential is also shown in Figs. 2(b) and 2(c) for comparison.

For a deeper analysis of the influence of the Coulomb potential on the PAD at different relative phases, we show both the one-dimensional measured and simulated angular distribution after integrating Figs. 3(a)-3(c) over $p_{\text {rel }}$, as shown in Fig. 3(d). Since the electron momentum distribution is central symmetrical in $0.5 \pi-1.5 \pi$ and $1.5 \pi-2 \pi(0 \pi)-0.5 \pi$, we only show the electron angular distribution in the range of $0.5 \pi-1.5 \pi$ in Fig. 3(d), which corresponds to PADs that are mainly distributed in the lower half of the $z-x$ plane. One can find that after including the Coulomb force, the simulated result (solid red line) is in quantitative agreement with the experiment (open circles). The two-hump distribution without the Coulomb potential (solid black line) disappears and only the peak around $180^{\circ}$ remains. The remaining peak is not located at the same position but is shifted to a smaller angle, and the yield of this peak is almost twice that of the peak without Coulomb potential.

In order to understand the origin of this angular distribution difference and also the subcycle dynamics of the photoelectron trajectory at different relative phases, we separated the electron angular distribution versus the $p_{\text {rel }}$ into four parts in terms of the electron birth time, as shown in Fig. 4. Here we only show the results when the electron tunneled out during the second and third quarter cycles [green and red parts in Fig. 1(a)], because these quarter cycles mainly contribute to the angular distribution in the lower half of the $z-x$ plane, which corresponds to the angular distribution in Fig. 3(d). In the results without Coulomb force, as illustrated in Figs. 4(a) and $4(\mathrm{c})$, it is clear that the angular distribution associated with the second and third quarter cycles contributes to one of the peaks in the solid black line of Fig. 3(d), respectively. After including the Coulomb potential, as shown in Figs. 4(b) and 4(d), however, one can clearly identify that more electrons are created in the third quarter cycle and the electron yield from the second quarter cycle is suppressed. In addition, the peak of the angular distribution from the second quarter cycle does not change, while it exhibits a small shift and is closer to $180^{\circ}$ from the third quarter cycle. This is the reason why we can only observe one slightly shifted peak in the solid red line of Fig. 3(d). More interestingly, the angular distribution related to the second quarter cycle is widely spread while the width does not change much for tunneling during the third quarter cycle. This indicates that the Coulomb force has a larger influence on the electrons that are released during the second quarter cycle compared to those from the third quarter cycle.

In a linearly polarized laser field the electrons that are ionized before a field maximum within a quarter of a laser cycle will be driven away from their origin by the laser field. We call them direct trajectories. However, those electrons ionized during a quarter cycle slightly after the field maximum will be driven back and can recollide with their parent ion. They are called recollision trajectories. In our two-color laser field, after including the Coulomb field the direct trajectories are strongly suppressed and their angular distribution is broader than that of the recollision trajectories at all relative 

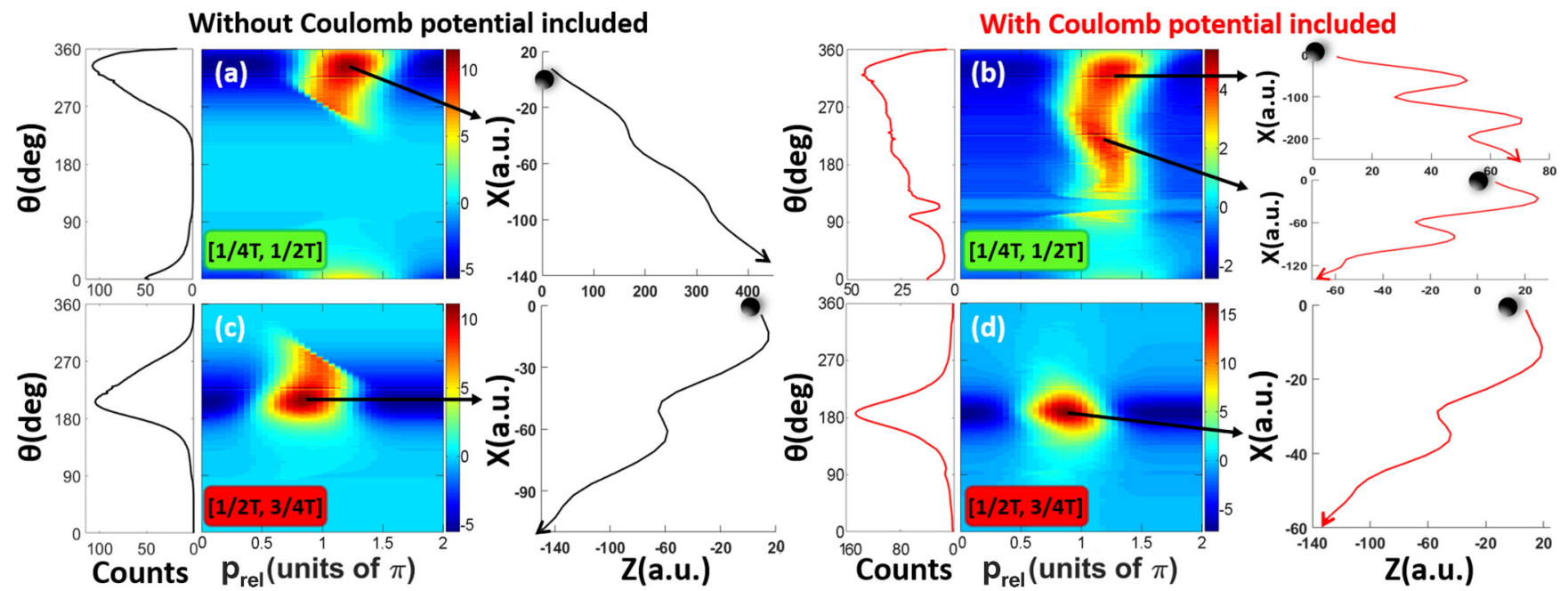

FIG. 4. CTMC simulated PADs as a function of the phase $p_{r e l}$ without (a), (c) and with (b), (d) the Coulomb potential for electron trajectories originating from the second (a), (b) and the third (c), (d) quarter cycle of the two-color field, as indicated in the panels. Left panel: Distribution of electron emission angle $\theta$ after integrating over the $p_{\text {rel }}$. Right panel: Typical trajectory corresponding to the yield maximum in the distribution. The black and red lines represent the results without and with inclusion of the Coulomb potential, respectively.

phases. Some typical trajectories corresponding to the yield maxima in Figs. 4(a)-4(d) are illustrated on the right-hand side. From the position-space analysis of trajectories one can clearly identify that with inclusion of the Coulomb force recollision trajectories do not change much, while the direct trajectories experience a larger distortion, and some of them are even driven back and recollide with the parent ion [see the trajectory at the bottom right of Fig. 2(b)]. This means the direct trajectories are more strongly affected by the ionic potential than the recollision trajectories, which is completely opposite to the electron behavior in monochromatic fields but in agreement with the results in OTC laser fields [24].

Based on the electron trajectory distribution, this suppression and stronger distortion of the direct trajectories can also be explained by the fact that the Coulomb field counteracts the laser field at the first few turning points and leads to a pulling back of the departing electron to some extent resulting in a reduced ionization probability. However, the Coulomb force does not distort recollision trajectory very much because it has the same direction as the laser field when the electron starts to return to the parent ion at the first turning point. As a result, it just increases the recollision velocity. Unlike the result in OTC laser fields, where the contribution from direct and recollision trajectories are still coupled in the momentum spectrum in the experiment, here we can directly distinguish them in the $p_{r e l}$-dependent photoelectron angular distribution, as seen in Fig. 3(a). The main distribution (dashed red box) only comes from the recollision trajectories, while the direct trajectories contribute to almost all of the remaining distribution. Thereby, the contribution from recollision and direct trajectories can be clearly distinguished and controlled by the relative phase. Since the direct trajectories have a stronger impact by the ionic potential, that means this special Coulomb effect can be directly visualized and controlled in the experiment utilizing this two-color field scheme.
We further investigate the influence of the Coulomb force on the photoelectron momentum distribution with respect to $p_{r e l}$. Figures 5(a)-5(c) and 5(e)-5(g) show the experimental and simulated results for transverse $\left(p_{x}\right)$ and longitudinal $\left(p_{z}\right)$ momentum distributions versus $p_{r e l}$, respectively. It is interesting that an initial $0.5 \pi p_{\text {rel }}$ difference between the $p_{x}$ and $p_{z}$ distributions versus $p_{\text {rel }}$ without considering the Coulomb potential [Figs. 5(b) and 5(f)] disappeared after including the Coulomb field [Figs. 5(c) and 5(g)], and the experimental results [Figs. 5(a) and 5(e)] confirm this as well. By comparing the CTMC results in Figs. 2(b) and 2(c), it becomes clear that this is caused by the Coulomb induced $\sim 45^{\circ}$ angle difference at each phase delay.

Moreover, both $p_{x}$ and $p_{z}$ distributions change after including the Coulomb potential. To illustrate this change more clearly, we integrate Figs. 5(a)-5(c) and 5(e) $-5(\mathrm{~g})$ over $p_{\text {rel }}$. The one-dimensional measured and simulated $p_{x}$ and $p_{z}$ distributions are shown in Figs. 5(d) and 5(h), respectively. Since the electron momentum distribution is central symmetrical in $0.5 \pi-1.5 \pi$ and $1.5 \pi-2 \pi(0 \pi)-0.5 \pi$, we also show here only the $p_{x}$ and $p_{z}$ distribution in the range of $0.5 \pi-1.5 \pi$ [dashed red box in Figs. 5(a)-5(c) and 5(e)-5(g)] in Figs. 5(d) and 5(h), which corresponds to PADs that are concentrated in the lower half of the $z-x$ plane. Comparing the simulated results without and with the Coulomb potential, one can find that $p_{x}$ becomes smaller after including the Coulomb potential while $p_{z}$ gets larger [see the arrows in Figs. 5(d) and 5(h)], and the simulated results including the Coulomb potential agree very well with the experimental results. This means that the Coulomb force has an opposite effect on $p_{x}$ and $p_{z}$.

To explore the cause of this opposite Coulomb effect on $p_{x}$ and $p_{z}$, we separated the $p_{x}$ and $p_{z}$ distribution with respect to the $p_{\text {rel }}$ into four parts in terms of the electron birth time as we did in the PAD spectra. The results are shown in Fig. 6. Here we also show only the results for electrons that get ionized in the second and third quarter cycles [green and red parts in Fig. 1(a)], because they mainly contribute to the PAD in the 

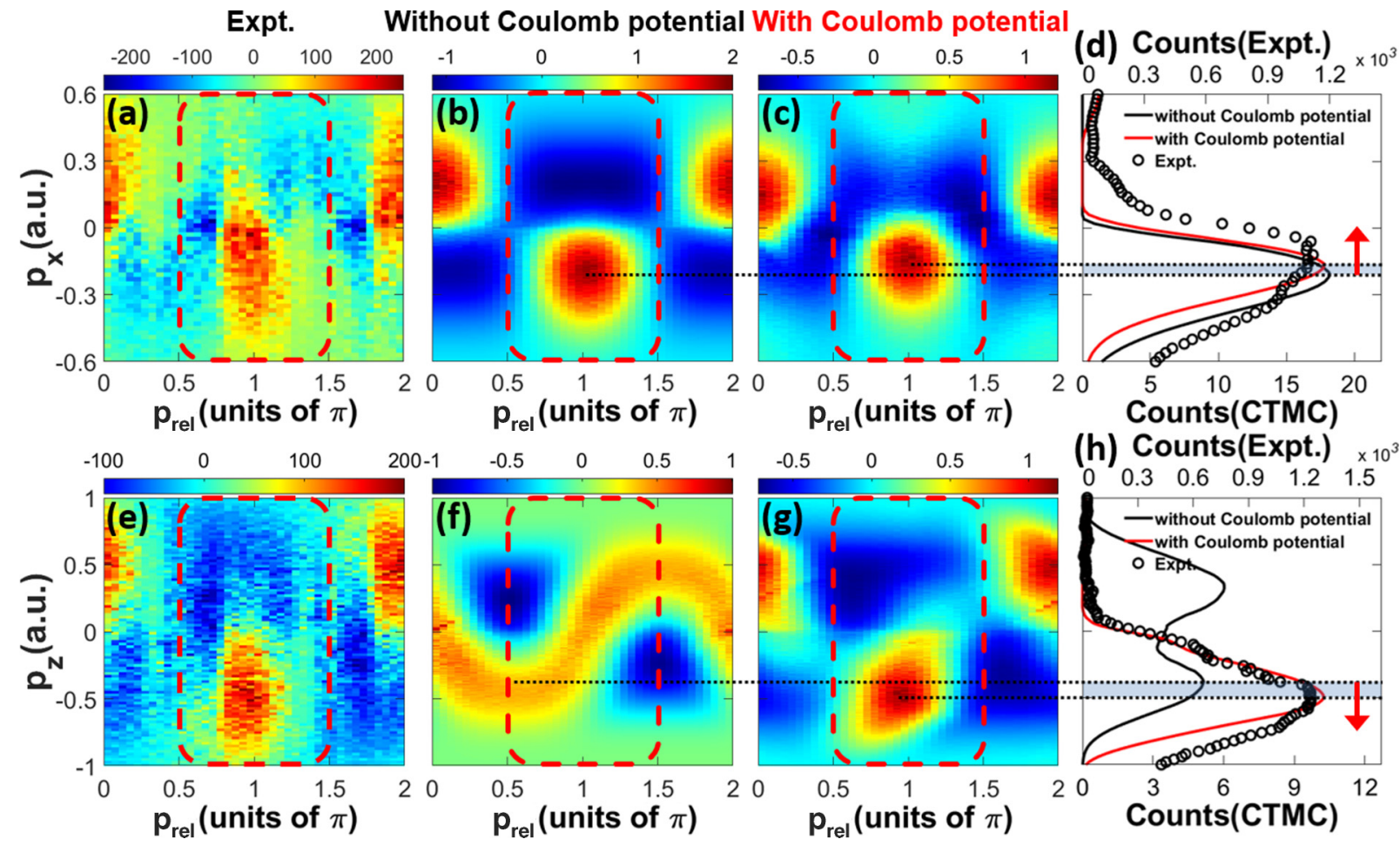

FIG. 5. The measured (a), (e) and simulated (b), (c), (f), (g) photoelectron momentum distribution for $p_{z}$ (a)-(c) and $p_{x}$ (e)-(g) as a function of the $p_{r e l}$. (b), (c), (f), (g) CTMC simulations without (b), (f) and with (c), (g) Coulomb potential included. (d), (h) The measured (open circles) and simulated (solid line) photoelectron $p_{z}(\mathrm{~d})$ and $p_{x}(\mathrm{~h})$ distribution after integrating over the $p_{\text {rel }}$ in the range from $0.5 \pi$ to $1.5 \pi$ [dashed red box in (a)-(c) and (e)-(g)]. The black and red lines represent the results without and with Coulomb potential, respectively. The red arrow indicates the direction of the peak position change from the black line to the red line.

lower half of the $z-x$ plane which corresponds to the $p_{x}$ and $p_{z}$ distribution in Figs. 5(d) and 5(h). For example, $p_{x}$ distribution versus $p_{\text {rel }}$ without Coulomb potential in the range of $0.5 \pi-1.5 \pi$ [the dashed red box in Fig. 5(b)] is separated into Figs. 6(a) and 6(d), which correspond to electrons released in the second and third quarter cycles, respectively. Similarly, the dashed red box in Fig. 5(c) is separated into Figs. 6(b) and 6(e), the dashed red box in Fig. 5(f) is divided into Figs. 6(g) and 6(j), and the dashed red box in Fig. 5(g) is separated into Figs. 6(h) and 6(k).

After integrating them over $p_{r e l}$, we get the $p_{x}$ and $p_{z}$ distribution as shown in the third column of Fig. 6. In Figs. 6(c) and 6(f), one can find that $p_{x}$ for both direct and recollision electrons becomes smaller after including the Coulomb potential. This is caused by the Coulomb focusing effect [37], but it plays a bigger role for the recollision electrons, as can be seen by the area of the shading in these two figures. This is because along the $x$ axis, there is only a weak transverse component of the blue circular field in this direction, and all electrons are driven farther and farther away from the parent ion along the $x$ direction. However, compared to the direct electrons, that feel a weaker and weaker Coulomb attraction when they leave the parent ion, the recollision electrons will feel a stronger Coulomb attraction as they approach the parent ion along the $z$ direction. However, the Coulomb force does not work the same way for the electrons in the $z$ direction as it does in the $x$ direction. As illustrated in Fig. 6(i), although the direct electrons have a smaller $p_{z}$ after including the Coulomb force because they still experience Coulomb focusing along the $z$ axis, their ionization probability is suppressed. For the recollision electrons, however, due to the strong linear red field along the $z$ axis, the Coulomb force always attracts them and accelerates them when they return to the parent ion. This not only increases the chance of a recollision with the parent ion but also produces a larger $p_{z}$, as shown in Fig. 6(1). As a result, the different influence of the Coulomb potential on direct and recollision electrons leads to a larger $p_{z}$ finally.

\section{CONCLUSION}

In conclusion, we have presented measurements of angleresolved photoelectron spectra for single ionization of $\mathrm{Ar}$ atoms in a two-color laser field with a linearly polarized 800 -nm field and a superimposed circularly polarized 400-nm field. The photoelectron angular distribution rotates at a nonconstant speed with the relative phase $p_{\text {rel }}$. It slows down around $p_{\text {rel }}=0.5 \pi+n \pi,(n=0,1,2 \cdots)$ and the rotation speed can be precisely controlled. Based on a semiclassical CTMC model, we separate the relative contributions of direct and recollision trajectories on the $p_{r e l}$-dependent angular distribution depending on the electron subcycle birth time. Our analysis shows that the direct trajectories are more affected by the Coulomb field than the recollision trajectories and we realized an experimental visualization that allows us to control this Coulomb effect in our two-color field scheme. Moreover, we find that the Coulomb effect on electrons in the transverse 
Without Coulomb potential With Coulomb potential
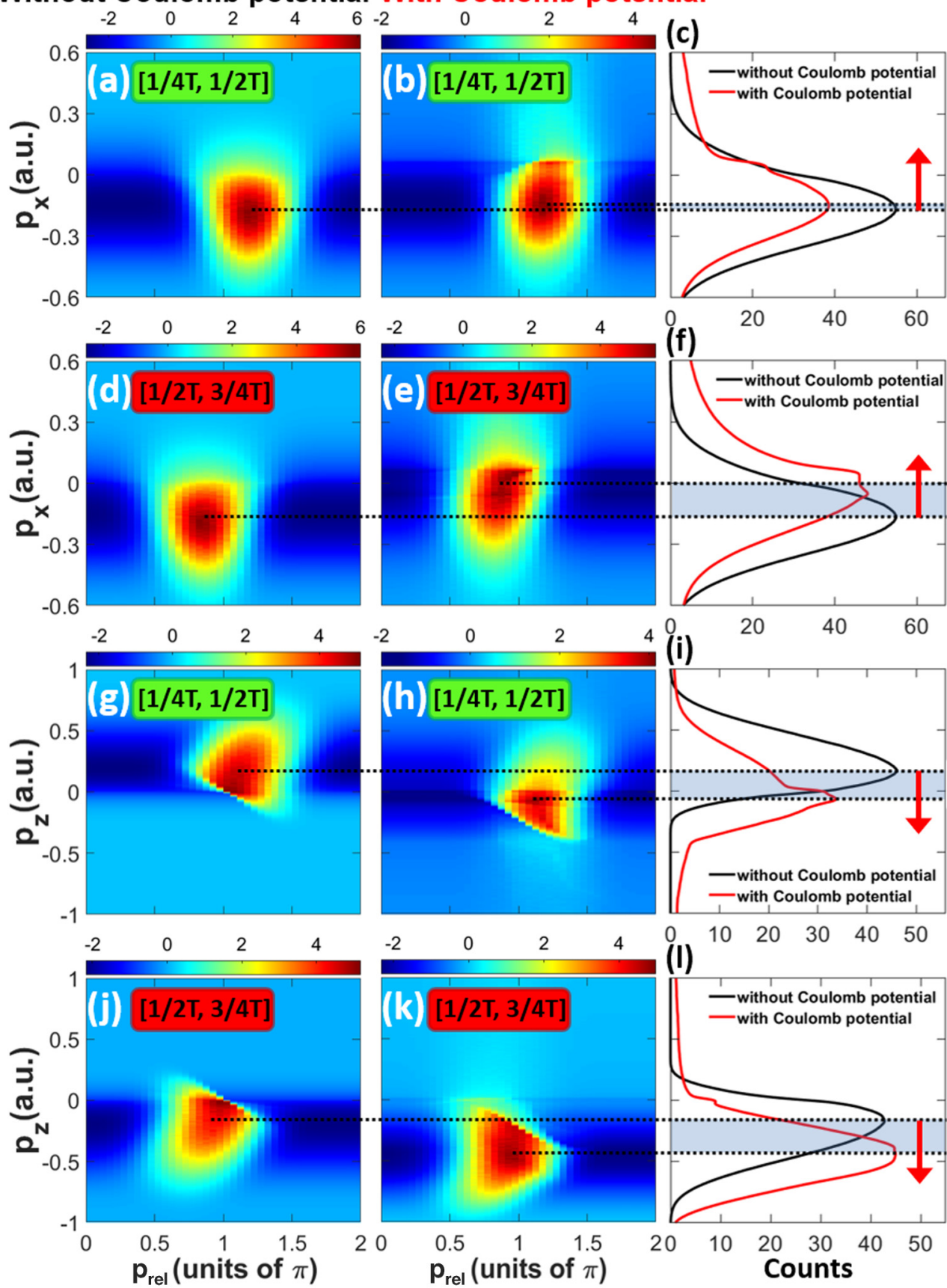

FIG. 6. CTMC simulated $p_{x}$ (a), (b), (d), (e) and $p_{z}(\mathrm{~g}),(\mathrm{h}),(\mathrm{j}),(\mathrm{k})$ distributions as a function of the $p_{\text {rel }}$ without (the first column) and with (the second column) Coulomb potential for electron trajectories originating from the second (a), (b), (g), (h) and the third (d), (e), (j), (k) quarter cycle of the two-color field, as indicated in the panels. (c), (f), (i), (l) The corresponding photoelectron $p_{x}$ (c), (f) and $p_{z}$ (i), (l) distributions after integrating over the $p_{r e l}$ in the range from $0.5 \pi$ to $1.5 \pi$. The black and red lines represent the results without and with Coulomb potential, respectively. The red arrow indicates the direction of the peak position change from the black line to the red line.

direction ( $x$ axis) is not the same as in the longitudinal direction ( $z$ axis), which results in an opposite change of momenta along the $p_{x}$ and $p_{z}$ directions. This kind of two-color field scheme could be used as a general tool for controlling the ultrafast dynamics of various atomic and molecular systems throughout the strong-field community.
[1] Progress in Ultrafast Intense Laser Science, edited by K. Yamanouchi, S. L. Chin, P. Agostini, and G. Ferrante (Springer,
Berlin, 2006); Advances of Atoms and Molecules in Strong Laser Fields, edited by Y. Liu (World Scientific, Singapore, 2015). 
[2] M. F. Kling, C. Siedschlag, A. J. Verhoef, J. I. Khan, M. Schultze, T. Uphues, Y. Ni, M. Uiberacker, M. Drescher, F. Krausz, and M. J. J. Vrakking, Science 312, 246 (2006).

[3] M. Kremer, B. Fischer, B. Feuerstein, V. L. B. de Jesus, V. Sharma, C. Hofrichter, A. Rudenko, U. Thumm, C. D. Schröter, R. Moshammer, and J. Ullrich, Phys. Rev. Lett. 103, 213003 (2009).

[4] B. Fischer, M. Kremer, T. Pfeifer, B. Feuerstein, V. Sharma, U. Thumm, C. D. Schröter, R. Moshammer, and J. Ullrich, Phys. Rev. Lett. 105, 223001 (2010).

[5] I. Znakovskaya, P. von den Hoff, G. Marcus, S. Zherebtsov, B. Bergues, X. Gu, Y. Deng, M. J. J. Vrakking, R. Kienberger, F. Krausz, R. de Vivie-Riedle, and M. F. Kling, Phys. Rev. Lett. 108, 063002 (2012)

[6] T. Rathje, A. M. Sayler, S. Zeng, P. Wustelt, H. Figger, B. D. Esry, and G. G. Paulus, Phys. Rev. Lett. 111, 093002 (2013).

[7] N. G. Kling, K. J. Betsch, M. Zohrabi, S. Zeng, F. Anis, U. Ablikim, B. Jochim, Z. Wang, M. Kübel, M. F. Kling, K. D. Carnes, B. D. Esry, and I. Ben-Itzhak, Phys. Rev. Lett. 111, 163004 (2013)

[8] H. Xu, T. Y. Xu, F. He, D. Kielpinski, R. T. Sang, and I. V. Litvinyuk, Phys. Rev. A 89, 041403(R) (2014).

[9] R. Kienberger, E. Goulielmakis, M. Uiberacker, A. Baltuska, V. Yakovlev, F. Bammer, A. Scrinzi, Th. Westerwalbesloh, U. Kleineberg, U. Heinzmann, M. Drescher, and F. Krausz, Nature (London) 427, 817 (2004).

[10] E. Goulielmakis, M. Uiberacker, R. Kienberger, A. Baltuska, V. Yakovlev, A. Scrinzi, Th. Westerwalbesloh, U. Kleineberg, U. Heinzmann, M. Drescher, and F. Krausz, Science 305, 1267 (2004).

[11] C. P. Hauri, W. Kornelis, F. W. Helbing, A. Couairon, A. Mysyrowicz, J. Bieglert, and U. Keller, Appl. Phys. B 79, 673 (2004).

[12] F. He, C. Ruiz, and A. Becker, Phys. Rev. Lett. 99, 083002 (2007).

[13] F. He, C. Ruiz, and A. Becker, J. Phys. B 41, 081003 (2008).

[14] K. P. Singh, F. He, P. Ranitovic, W. Cao, S. De, D. Ray, S. Chen, U. Thumm, A. Becker, M. M. Murnane, H. C. Kapteyn, I. V. Litvinyuk, and C. L. Cocke, Phys. Rev. Lett. 104, 023001 (2010).

[15] G. Sansone, F. Kelkensberg, J. F. Pérez-Torres, F. Morales, M. F. Kling, W. Siu, O. Ghafur, P. Johnsson, M. Swoboda, E. Benedetti, F. Ferrari, F. Lépine, J. L. Sanz-Vicario, S. Zherebtsov, I. Znakovskaya, A. L'Huillier, M. Yu. Ivanov, M. Nisoli, F. Martín, and M. J. J. Vrakking, Nature (London) 465, 763 (2010).

[16] X. Xie, S. Roither, D. Kartashov, E. Persson, D. G. Arbó, L. Zhang, S. Gräfe, M. S. Schöffler, J. Burgdörfer, A. Baltuška, and M. Kitzler, Phys. Rev. Lett. 108, 193004 (2012).
[17] D. G. Arbó, S. Nagele, X.-M. Tong, X. Xie, M. Kitzler, and J. Burgdörfer, Phys. Rev. A 89, 043414 (2014).

[18] D. G. Arbó, C. Lemell, S. Nagele, N. Camus, L. Fechner, A. Krupp, T. Pfeifer, S. D. López, R. Moshammer, and J. Burgdörfer, Phys. Rev. A 92, 023402 (2015).

[19] S. Luo, M. Li, H. Xie, P. Zhang, S. Xu, Y. Li, Y. Zhou, P. Lan, and P. Lu, Phys. Rev. A 96, 023417 (2017).

[20] A. D. Bandrauk and S. Chelkowski, Phys. Rev. Lett. 84, 3562 (2000).

[21] D. Ray, F. He, S. De, W. Cao, H. Mashiko, P. Ranitovic, K. P. Singh, I. Znakovskaya, U. Thumm, G. G. Paulus, M. F. Kling, I. V. Litvinyuk, and C. L. Cocke, Phys. Rev. Lett. 103, 223201 (2009).

[22] K. J. Betsch, D. W. Pinkham, and R. R. Jones, Phys. Rev. Lett. 105, 223002 (2010).

[23] W. Zhang, H. Li, K. Lin, P. Lu, X. Gong, Q. Song, Q. Ji, J. Ma, H. Li, H. Zeng, F. He, and J. Wu, Phys. Rev. A 96, 033405 (2017).

[24] L. Zhang, X. Xie, S. Roither, D. Kartashov, Y. Wang, C. Wang, M. Schöffler, D. Shafir, P. B. Corkum, A. Baltuška, I. Ivanov, A. Kheifets, X. Liu, A. Staudte, and M. Kitzler, Phys. Rev. A 90, 061401(R) (2014).

[25] L. Zhang, X. Xie, S. Roither, Y. Zhou, P. Lu, D. Kartashov, M. Schöffler, D. Shafir, P. B. Corkum, A. Baltuška, A. Staudte, and M. Kitzler, Phys. Rev. Lett. 112, 193002 (2014).

[26] Y. Zhou, C. Huang, A. Tong, Q. Liao, and P. Lu, Opt. Express 19, 2301 (2011).

[27] X. Gong, P. He, Q. Song, Q. Ji, H. Pan, J. Ding, F. He, H. Zeng, and J. Wu, Phys. Rev. Lett. 113, 203001 (2014).

[28] Q. Song, P. Lu, X. Gong, Q. Ji, K. Lin, W. Zhang, J. Ma, H. Zeng, and J. Wu, Phys. Rev. A 95, 013406 (2017).

[29] K. Lin, X. Gong, Q. Song, Q. Ji, W. Zhang, J. Ma, P. Lu, H. Pan, J. Ding, H. Zeng, and J. Wu, J. Phys. B 49, 025603 (2016).

[30] M. Han, P. Ge, Y. Shao, Q. Gong, and Y. Liu, Phys. Rev. Lett. 120, 073202 (2018).

[31] Y. Fang, C. He, M. Han, P. Ge, X. Yu, X. Ma, Y. Deng, and Y. Liu, Phys. Rev. A 100, 013414 (2019).

[32] G. L. Yudin and M. Y. Ivanov, Phys. Rev. A 64, 013409 (2001).

[33] R. Dörner, V. Mergel, O. Jagutzki, L. Spielberger, J. Ullrich, R. Moshammer, and H. Schmidt-Böcking, Phys. Rep. 330, 95 (2000).

[34] L. D. Landau and E. M. Lifshitz, in Quantum Mechanics (Pergamon Press, Oxford, 1977), p. 293.

[35] M. V. Ammosov, N. B. Delone, and V. P. Krainov, Zh. Eksp. Teor. Fiz. 91, 2008 (1986) [Sov. Phys. JETP 64, 1191 (1986)].

[36] N. B. Delone and V. P. Krainov, J. Opt. Soc. Am. B 8, 1207 (1991).

[37] T. Brabec, M. Y. Ivanov, and P. B. Corkum, Phys. Rev. A 54, R2551(R) (1996). 